\title{
COMPARISON OF LANDSAT-8 AND SENTINEL-2 DATA FOR CLASSIFICATION OF RABI CROPS OVER KARNATAKA, INDIA
}

\author{
Subir Paul ${ }^{1, *}$, D. Nagesh Kumar ${ }^{1,2}$ \\ ${ }^{1}$ Department of Civil Engineering, Indian Institute of Science, Bengaluru-560012, India - subir.paul.iisc@gmail.com \\ ${ }^{2}$ Interdisciplinary Centre for Water Research, Indian Institute of Science, Bengaluru-560012, India - nagesh@iisc.ac.in
}

Commission III, WG III/10

KEY WORDS: Crop Classification, Landsat-8, Sentinel-2, Normalized Difference Indices, Partial Informational Correlation, Support Vector Machine Classifier

\begin{abstract}
:
Classification of crops is very important to study different growth stages and forecast yield. Remote sensing data plays a significant role in crop identification and condition assessment over a large spatial scale. Importance of Normalized Difference Indices (NDIs) along with surface reflectances of remotely sensed spectral bands have been evaluated for classification of eight types of Rabi crops utilizing the Landsat- 8 and Sentinel-2 datasets and performances of both the satellites are compared. Landsat- 8 and Sentinel-2A images are acquired for the location of crops and seven and nine spectral bands are utilized respectively for the classification. Experiments are carried out considering the different combinations of surface reflectances of spectral bands and optimal NDIs as features in support vector machine classifier. Optimal NDIs are selected from the set of ${ }^{7} \mathrm{C}_{2}$ and ${ }^{9} \mathrm{C}_{2}$ NDIs of Landsat- 8 and Sentinel$2 \mathrm{~A}$ datasets respectively using the partial informational correlation measure, a nonparametric feature selection approach. Few important vegetation indices (e.g. enhanced vegetation index) are also experimented in combination with the surface reflectances and NDIs to perform the crop classification. It has been observed that combination of surface reflectances and optimal NDIs can classify the crops more efficiently. The average overall accuracy of $80.96 \%$ and $88.16 \%$ are achieved using the Landsat- 8 and Sentinel-2A datasets respectively. It has been observed that all the crop classes except Paddy and Cotton achieve producer accuracy and user accuracy of more than $75 \%$ and $85 \%$ respectively. This technique can be implemented for crop identification with adequate accessibility of crop information.
\end{abstract}

\section{INTRODUCTION}

Crop classification and identification have great significance in assessing the soil and water requirements and the growing habit of different crops. Cropland mapping and monitoring are very important for estimation of potential harvesting, agricultural field management (Sonobe et al. 2018), food production and sustainable natural resources management (Belgiu and Csillik 2018). Hence, it is crucial for Indian economy where agricultural sector provides $50 \%$ of total employment. Traditional ways of obtaining the crop information through census or field survey are very inefficient and time-consuming. However remotely sensed satellite images effectual in obtaining global information. Remote sensing data are very important and effective tool for monitoring earth surface features and collecting the information about spatio-temporal variability of the land surface (Jia et al. 2014). Remote sensing is the only existing practical way of continuous land cover information generation over large areas (Momeni et al. 2016).

Crop type discrimination is a major challenge for small agriculture plots with diversified cropping systems. However, with the free availability of high resolution remote sensing images at local or national or global scales (Forkuor et al. 2018), different types of crop can be identified at very fine spatial resolution. The new generation of multispectral sensors carried on the Landsat- 8 and Sentinel-2 satellites offer continuous spatial and temporal monitoring applications (Mandanici and Bitelli 2016). Landsat-8 and Sentinel-2 are the most widely used optical remote sensing satellites since these satellite images are freely available globally. Different studies had evaluated the performances of Landsat- 8 and Sentinel-2 data based on diversified applications viz. detection of greenhouse (Novelli et al. 2016), estimation of forest canopy cover and Leaf Area Index (LAI) (Korhonen et al. 2017), detection of $\mathrm{C} 3$ and $\mathrm{C} 4$ grass species (Shoko and Mutanga 2017), land use land cover mapping (Forkuor et al. 2018), characterization of reflectance and Normalized Difference Vegetation Index (NDVI) (Zhang et al. 2018) etc. and in most of the applications it was evident that Sentinel-2 was performing better because of its improved spatial and spectral capabilities. However, none of the studies were undertaken in India till date to evaluate the performance of Landsat- 8 and Sentinel-2 datasets in crop classification.

NDVI and Enhanced Vegetation Index (EVI) are most extensively used Vegetation Indices (VIs) for vegetation monitoring. Different Normalized Difference Indices (NDIs) viz. NDVI, Green NDVI and Normalized Difference Red Edge index (NDRE) of RapidEye imagery have been investigated for different applications such as early stress detection (Eitel et al. 2011) and crop classification (Ustuner et al. 2014). All possible combinations of NDIs, which are obtained from the surface reflectances of spectral bands have not been evaluated yet for the purpose of crop classification. These NDIs can provide significant information for efficient crop classification. Therefore, the objectives of this study are as follows: firstly, checking the significance of NDIs and important VIs along with surface reflectances of spectral bands for crop classification; and secondly, comparison of the Landsat- 8 and Sentinel-2 datasets for crop classification. These two objectives are basically dealing with the qualities of the data or features or variables, however, use of efficient classification algorithm is also important (Sonobe et al. 2018).

\footnotetext{
* Corresponding author
} 
Support Vector Machine (SVM) is the most widely used nonparametric classifier without any assumptions about the underlying data distribution. Though performances of different classifiers mostly depend on the used dataset (Paul and Kumar 2018), numerous studies had demonstrated that SVM is the most promising machine learning classifier for land use land cover classification (Jia et al. 2014; Mountrakis et al. 2011; Pal and Mather 2005). Hence, SVM classifier is adopted here for crop classification.

\section{MATERIALS AND METHODS}

\subsection{Study Area and Field Data}

This study is carried out over the Karnataka state of India. The crop information (i.e. ground-truth data) was collected over few locations of Karnataka (which are shown in Figure 1) by the Mahalanobis National Crop Forecast Centre (MNCFC), New Delhi, India. The crop details, provided by the MNCFC, were mostly collected during the December month of 2015 (i.e. Rabi season of 2015-16). These ground-truths were available for eight types of crops viz. Bengalgram, Chilli, Cotton, Groundnut, Jowar, Paddy, Toor and Wheat.

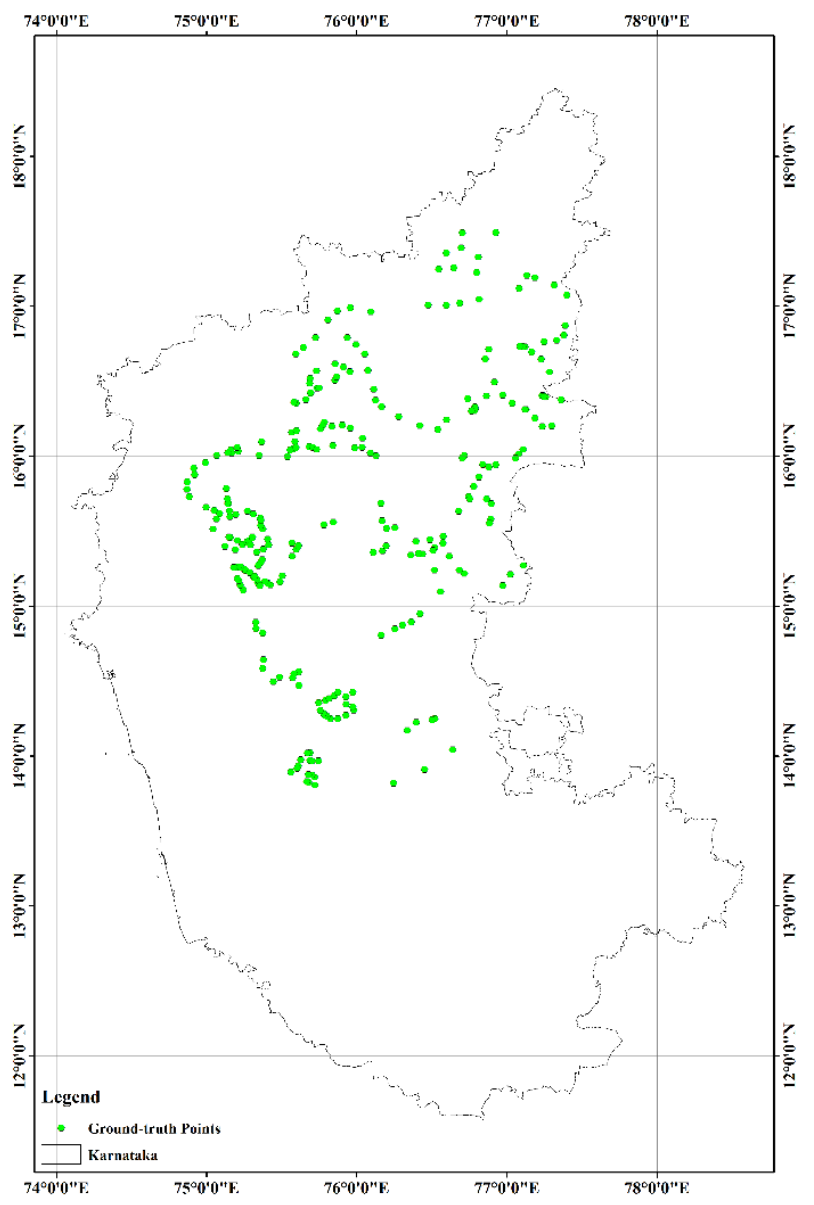

Figure 1. Locations of ground-truth points (crop information) over Karnataka State

\subsection{Satellite Data}

2.2.1 Landsat-8: Landsat-8 was launched on 11 February 2013 with a multispectral Operational Land Imager (OLI) sensor. Atmospherically corrected surface reflectances of multispectral bands of Landsat-8 (Landsat-8 OLI C1 Level-2 product), which are derived using the Landsat- 8 Surface Reflectance Code (LaSRC) algorithm (Vermote et al. 2016), are freely available and can be downloaded from the United States Geological Survey (USGS) website (https://earthexplorer.usgs.gov/). The wavelength ranges of seven spectral bands of Landsat-8 OLI sensor are presented in Table 1. The details about the collection of cloud-free Landsat- 8 images, covering the study area, are presented in Table 2.

\begin{tabular}{|c|c|c|}
\hline \multirow{2}{*}{ Spectral bands } & \multicolumn{2}{|c|}{ Wavelength (nm) range } \\
\cline { 2 - 3 } & Landsat-8 & Sentinel-2 \\
\hline Band 1 & $435-451$ & - \\
\hline Band 2 & $452-512$ & $448-546$ \\
\hline Band 3 & $533-590$ & $537.5-582.5$ \\
\hline Band 4 & $636-673$ & $646-684$ \\
\hline Band 5 & $851-879$ & $694.5-713.5$ \\
\hline Band 6 & $1566-1651$ & $731-749$ \\
\hline Band 7 & $2107-2294$ & $768-796$ \\
\hline Band 8a & - & $848.5-881.5$ \\
\hline Band 11 & - & $1542.5-1685.5$ \\
\hline Band 12 & - & $2081-2323$ \\
\hline
\end{tabular}

Table 1: Details of spectral bands of both satellites

\begin{tabular}{|c|c|}
\hline Image acquisition date & path/row \\
\hline $22-12-2015$ & $145 / 47,145 / 48,145 / 49,145 / 50$, \\
& $145 / 51,145 / 52$ \\
\hline $24-12-2015$ & $143 / 51$ \\
\hline $29-12-2015$ & $146 / 48,146 / 49,146 / 50,146 / 51$ \\
\hline $31-12-2015$ & $144 / 51$ \\
\hline
\end{tabular}

Table 2: Details of acquired Landsat-8 images

2.2.2 Sentinel-2: The Sentinel-2A and $2 B$ satellites were launched on 23 June, 2015 and 7 March, 2017 respectively. Sentinel-2 MultiSpectral Instrument (MSI) Level-1C products are freely available for download at the Sentinels Scientific Data Hub website (https://scihub.copernicus.eu/dhus/\#/home). This data contains the spectral responses of 13 bands, spanning from visible to ShortWave InfraRed (SWIR) at $10 \mathrm{~m}$ (4 bands), $20 \mathrm{~m}$ ( 6 bands) and $60 \mathrm{~m}$ ( 3 bands) spatial resolution. The Sentinel-2 images can be atmospherically corrected using the European Space Agency's (ESA) Sen2Cor atmospheric correction toolbox (Muller-Wilm et al. 2013), which is an inbuilt algorithm within the SentiNel Application Platform (SNAP) tool version 6.0 to produce the Level-2A (L2A) products. The atmospherically corrected L2A product contains surface reflectances of 9 spectral bands which are utilized in this study and the details about these spectral bands are presented in Table 1. Sentinel-2A satellite data are used in this study, since Sentinel-2B was not launched during the time-period of accessible ground-truth collection. The details about the collection of cloud-free Sentinel-2A images, covering the study area, are shown in Table 3.

\begin{tabular}{|c|c|}
\hline Image acquisition date & Number of tiles \\
\hline $21-12-2015$ & 9 \\
\hline $24-12-2015$ & 27 \\
\hline $29-12-2015$ & 2 \\
\hline
\end{tabular}

Table 3: Details of Sentinel-2A image acquisition 


\subsection{Data Pre-processing}

Sentinel-2A MSI images are atmospherically corrected to obtain the L2A products, which offer the surface reflectances of different spectral bands. These L2A products are derived at spatial resolution of $20 \mathrm{~m}$. Hence, Sentinel-2A images are upscaled to the spatial resolution of Landsat- 8 (i.e. $30 \mathrm{~m}$ ) to compare the performances of the datasets of two satellites in the same spatial-scale. All the satellite images are co-registered to the same projection system (UTM/WGS84).

The areas of the crop fields are digitized corresponding to the provided Lat/Lon of the ground-truth locations with the help of Google Earth and ArcGIS software. The pixels are identified corresponding to each crop field area from both the satellite images. Number of pixels obtainable for each crop type is mentioned in Table 4.

\begin{tabular}{|c|c|c|c|c|}
\hline $\begin{array}{c}\text { Sl. } \\
\text { No. }\end{array}$ & Crop type & $\begin{array}{c}\text { Training } \\
\text { pixels }\end{array}$ & $\begin{array}{c}\text { Testing } \\
\text { pixels }\end{array}$ & $\begin{array}{c}\text { Total } \\
\text { pixels }\end{array}$ \\
\hline 1 & Bengalgram & 106 & 108 & 214 \\
\hline 2 & Chilli & 124 & 126 & 250 \\
\hline 3 & Cotton & 19 & 18 & 37 \\
\hline 4 & Groundnut & 5 & 4 & 9 \\
\hline 5 & Jowar & 215 & 216 & 431 \\
\hline 6 & Paddy & 4 & 6 & 10 \\
\hline 7 & Toor & 34 & 36 & 70 \\
\hline 8 & Wheat & 7 & 8 & 15 \\
\hline
\end{tabular}

Table 4: Details of the number of pixels for different type of crops

\subsection{Methodology}

Two different experiments are attempted for classification of eight types of Rabi crops. In the first experiment, only surface reflectances of spectral bands are used as features which is the most conventional way of classification. In the second experiment NDIs and important VIs are utilized along with the surface reflectances of spectral bands as inputs in the classifier model. There are ${ }^{7} \mathrm{C}_{2}$ and ${ }^{9} \mathrm{C}_{2}$ possible combinations of NDIs to be evaluated from the Landsat-8 and Sentinel-2A images respectively. NDVI, Green NDVI, NDRE and EVI are few important VIs, among which all VIs except EVI come under the group of NDIs. The number of input variables is increased by considering all these NDIs and VIs which can introduce the issue of data redundancy and simply increase the complexity of the classifier algorithm. Therefore, a non-parametric conditional dependency measure PIC has been employed to identify the most useful NDIs and VIs for crop classification. In the second classification experiment, PIC is also applied to the surface reflectances of spectral bands to check the importance of these surface reflectance information in classifying the eight types of crops. The classification methodology of this experiment is presented via a flowchart in Figure 2.

\subsection{Partial Informational Correlation}

Partial Information (PI) is a non-parametric and information theory based measure of conditional dependence, which can be used to identify predictor variables (Sharma and Mehrotra 2014). PI can be transformed to a scale of $0-1$, where 0 and 1 represent no conditional correlation and perfect conditional correlation respectively regardless of any distributional assumptions about the variables. The rescaled dependence measure is called PIC. Further details about the PIC measure can be found in Sharma and Mehrotra (2014). In this study,
PICs are calculated between the crop class types and input variables (or features) utilizing the NPRED package (Sharma et

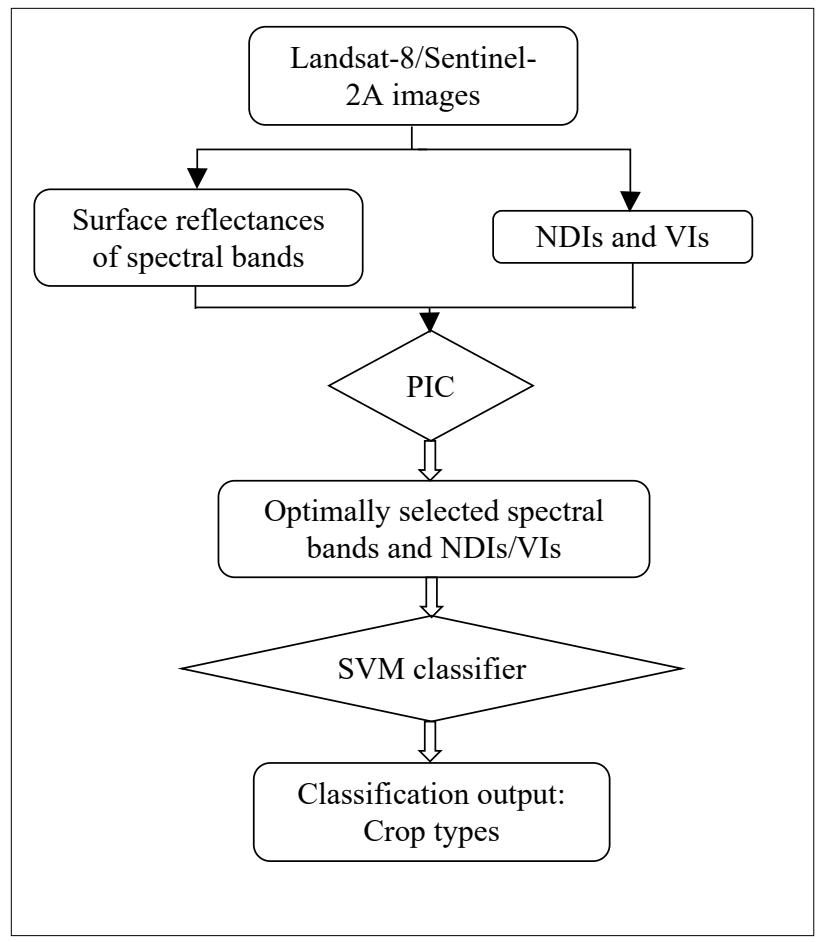

al. 2016) in open-source R-software.

Figure 2. Flowchart of second classification experiment

\subsection{Support Vector Machine Classifier}

The SVM is a supervised non-probabilistic and non-parametric statistical learning technique (Mountrakis et al. 2011). SVM was initially designed for binary classification problems and in its simplest form, it could only classify the linearly separable classes (Pal and Mather 2005). Error Correcting Output Codes (ECOC) model is used to solve the multiclass classification problem by dissolving it into a set of binary classification problems. Further, to deal with the nonlinear datasets, different kernel functions (e.g. Gaussian, polynomial) can be used in the SVM algorithm. In this study, Gaussian kernel function, which is mostly considered in SVM algorithm (Shao and Lunetta 2012), is employed to classify nonlinear dataset and the parameters of the classifier are evaluated using Bayesian optimization technique.

\subsection{Experiment Setup}

The classification approaches are evaluated in MATLAB environment (version 9.4, 64-bit), with Intel(R) Core(TM) i54460 CPU@3.20 GHz Processor, 16.00 GB memory (RAM) and NVIDIA GeForce 210 graphics card. All the experiments are carried out considering $50 \%$ of the labelled pixels from each crop class as training dataset, and rest of the labelled pixels as testing dataset [Table 4]. 10 trials are performed considering 10 sets of random sampling for partitioning of the training and testing data. Thereafter mean and Standard Deviation (SD) of the performance evaluation measures are calculated from the results of 10 sets of testing data and reported for each experiment [Table 5]. Overall Accuracy (OA), kappa coefficient (k) and Average Accuracy (AA) are considered as performance evaluation measures. User Accuracy (UA) and Producer Accuracy (PA) are calculated for performance evaluation of 
each crop. McNemar test is performed to check the statistical significance of the differences (i.e. increase or decrease) in performances of the two different classification experiments.

\section{RESULTS AND DISCUSSION}

\subsection{Spectral Curves of Different Crops}

Average spectral response curves are created by averaging the surface reflectances of all the spectral bands. The spectral response of each band is calculated considering the average response of all the pixels of each crop. The average spectral response curves derived from the Landsat- 8 and Sentinel-2A datasets are presented in Figure 3 and 4 respectively. The error bars of the spectral curves are representing the SD of the surface reflectances. It has been observed in Figure 3 and 4 that spectral responses of different crops are more distinguishable in the NIR and SWIR wavelength ranges compared to visible range.

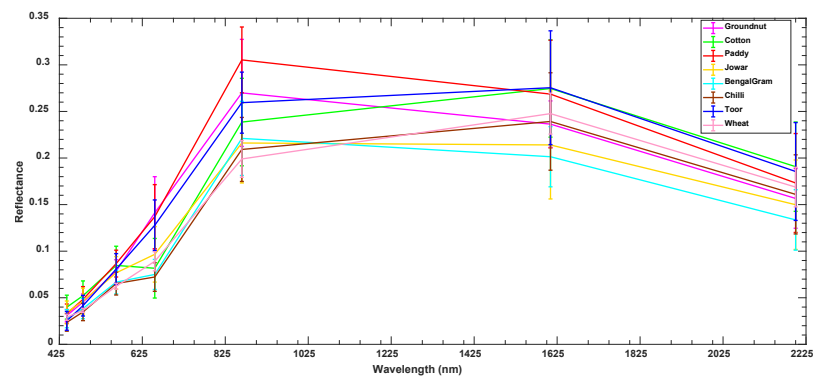

Figure 3. Average spectral response curves of each crop derived from Landsat- 8 data

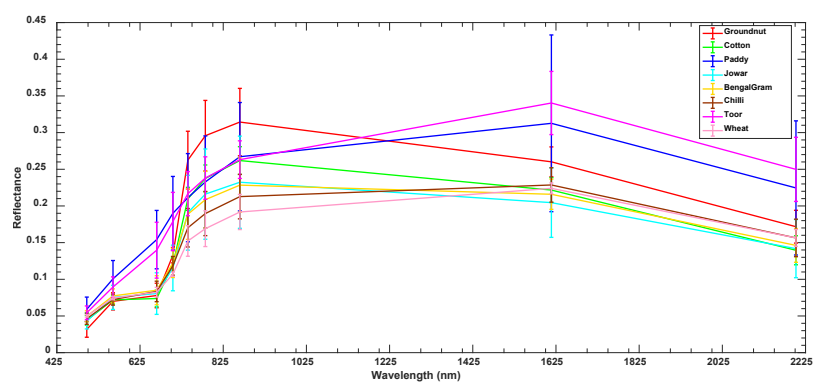

Figure 4. Average spectral response curves of each crop derived from Sentinel-2A data

\subsection{PIC based Feature Selection}

PIC-based feature selection approach has been used in this study for crop classification by identifying the salient features from surface reflectances of spectral bands, NDIs and VIs.

In case of Landsat- 8 data, 6 NDIs and EVI are selected optimally by employing the PIC-based feature selection approach on the set of all NDIs and EVI dataset. These 6 NDIs are calculated from the band combinations of $7 \& 2,6 \& 4,6 \& 1$, $5 \& 2,3 \& 2$ and $5 \& 4$ (i.e. NDVI). All the 7 spectral bands are identified as features, and therefore total 14 features are utilized in the SVM classifier for classification of crops.

In case of Sentinel-2A data, 5 spectral bands are optimally selected among the 9 bands and 4 NDIs are selected from the set of ${ }^{9} \mathrm{C}_{2}$ combinations of NDIs and EVI. The selected spectral bands are Band 2 (Blue), 3 (Green), 5 (Red-edge), 11 (SWIR) and 12 (SWIR). The identified NDIs are calculated from the spectral band combinations of $11 \& 2,5 \& 3,12 \& 2$ and $7 \& 4$ (i.e. NDVI). It is evident from the optimal feature selection process that spectral responses in the blue, red-edge and SWIR wavelength ranges are more significant and suitable for classification of these crops.

\subsection{Comparison of Classification Performances}

In the first experiment, classification performances are compared only utilizing the surface reflectances of spectral bands of Landsat- 8 and Sentinel-2A datasets and produce average OA of $79.94 \%$ and $87.41 \%$ respectively. In the second experiment, NDIs and VIs are considered along with surface reflectances of spectral bands, where optimal features are selected employing the PIC measure for classification, and yield average OA of $80.96 \%$ and $88.16 \%$ respectively with the Landsat-8 and Sentinel-2A datasets. The classification performances of both the experiments considering Landsat- 8 and Sentinel-2A datasets are presented in Table 5. It has been observed that the Sentinel-2A dataset are able to classify the eight types of Rabi crops more efficiently with statistically improved performance compared to the Landsat- 8 dataset in case of both the experiments. The main possible reason for this improvement in the performances with the Sentinel-2A dataset is the availability of red-edge spectral bands. The efficacy of red-edge spectral bands of different sensors are analysed in vegetation related earlier studies (Eitel et al. 2011; Korhonen et al. 2017; Shoko and Mutanga 2017; Ustuner et al. 2014) and proven to be very efficient for vegetation monitoring and classification. The improvements in the average classification performances by introducing the NDIs as features in the classifier model are insignificant for both the satellite datasets but in case of $50 \%$ of trials (i.e. 5 out of 10 trials) statistically improved performances are observed.

Another experiment is performed with the Sentinel-2A dataset by considering only optimally selected NDIs and important VIs (i.e. NDVI, Green NDVI, NDRE and EVI) as features for crop classification, which yield an average OA of $83.68 \%$. This experiment proves that neither surface reflectances nor NDIs and VIs alone are efficient enough to achieve the optimal classification performance.

\begin{tabular}{|c|c|c|c|}
\hline Features & OA (\%) & k & AA (\%) \\
\hline \multicolumn{4}{|c|}{ Landsat-8 } \\
\hline Only spectral & $79.94 \pm$ & $0.7194 \pm$ & $63.83 \pm$ \\
bands & 1.56 & 0.0190 & 6.57 \\
\hline Optimally & $80.96 \pm$ & $0.7341 \pm$ & $65.67 \pm$ \\
selected spectral & 1.59 & 0.0209 & 6.93 \\
bands and NDIs & \multicolumn{4}{|c|}{ Sentinel-2 } \\
\hline \multicolumn{4}{|c|}{} \\
\hline Only spectral & $87.41 \pm$ & $0.8246 \pm$ & $79.93 \pm$ \\
bands & 1.93 & 0.0272 & 4.79 \\
\hline Optimally & $\mathbf{8 8 . 1 6} \pm$ & $\mathbf{0 . 8 3 4 9} \pm$ & $\mathbf{8 1 . 7 9 \pm}$ \\
selected spectral & $\mathbf{1 . 8 4}$ & $\mathbf{0 . 0 2 6 3}$ & $\mathbf{2 . 3 5}$ \\
bands and NDIs &
\end{tabular}

Table 5: Crop classification performances for different experiments

\subsection{Classification Performance of Each Crop}

Classification performance of each crop can be evaluated in terms of UA and PA, which are calculated from a confusion matrix. Confusion matrix reports the number of pixels correctly classified and misclassified. The confusion matrix has been created from the performance of Sentinel-2A dataset utilizing the optimally selected spectral bands and NDIs [Table 6]. This is generated based on the performance of testing dataset of a 
single trial. It is observed that Cotton and Paddy are classified very poorly with $\mathrm{PA}$ of $66.67 \%$ and $16.67 \%$ respectively. The pixels, belonging to Paddy, are misclassified as Jowar and Toor. Paddy crop has very few pixels which are not sufficient enough to train the classifier efficiently, hence the performance is poorer compared to the other crops. On the contrary, Groundnut and Wheat also have very few pixels but their PAs are comparatively better. Hence, the possible reason of Cotton's poor performance can be non-uniformity in the spectral responses of Cotton pixels. The UA of all the crop types except Cotton and Paddy is more than $85 \%$. This issue of misclassification of the crops can be overcome by using an adequate number of pixels per class to train the classifier model.

\begin{tabular}{|c|c|c|c|c|c|c|c|c|c|c|}
\hline & & \multicolumn{8}{|c|}{ Predicted (Output) class } & \\
\hline & Crop & Groundnut & Cotton & Paddy & Jowar & Bengalgram & Chilli & Toor & Wheat & PA (\%) \\
\hline \multirow{8}{*}{ 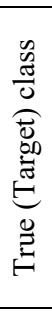 } & Groundnut & 3 & 0 & 0 & 1 & 0 & 0 & 0 & 0 & 75 \\
\hline & Cotton & 0 & 12 & 0 & 1 & 2 & 3 & 0 & 0 & 66.67 \\
\hline & Paddy & 0 & 0 & 1 & 3 & 0 & 0 & 2 & 0 & 16.67 \\
\hline & Jowar & 0 & 0 & 0 & 200 & 10 & 3 & 2 & 1 & 92.59 \\
\hline & Bengalgram & 0 & 1 & 0 & 3 & 98 & 6 & 0 & 0 & 90.74 \\
\hline & Chilli & 0 & 4 & 0 & 0 & 4 & 118 & 0 & 0 & 93.65 \\
\hline & Toor & 0 & 1 & 1 & 2 & 1 & 1 & 30 & 0 & 83.33 \\
\hline & Wheat & 0 & 0 & 0 & 0 & 0 & 1 & 0 & 7 & 87.50 \\
\hline & UA (\%) & 100 & 66.67 & 50 & 95.24 & 85.22 & 89.39 & 88.23 & 87.50 & OA: 89.85 \\
\hline
\end{tabular}

Table 6: Confusion matrix created from the best performing result of Sentinel-2A dataset

\section{CONCLUSIONS}

Landsat- 8 and Sentinel-2A datasets are compared in this study for classification of eight types of Rabi crops. Basically, two experiments are employed for classification of crops, I) surface reflectances of spectral bands and II) NDIs and VIs along with the surface reflectances of spectral bands are used as features in the SVM classifier. In both experiments, Sentinel-2A dataset provides better crop classification accuracies compared to the Landsat- 8 dataset. This improvement is mainly observed because of the presence of red-edge spectral bands in Sentinel2A dataset. Classification performance of each crop is evaluated from confusion matrix and it is observed that most of the crops are classified accurately except Cotton and Paddy, because of unavailability of pixels with uniform spectral responses and adequate number of pixels respectively. The UA and PA of each crop can further be improved with the availability of more number of pixels for training of SVM classifier.

This study can be further extended to state and country scales for cropland mapping with the availability of adequate information about all crop types. The current available remote sensing images from Sentinel-2A and 2B satellites can provide more frequent multi-temporal datasets during different crop seasons, which is beneficial in monitoring the growth-stages of different crops with the availability of proper ground-truth information.

\section{ACKNOWLEDGMENT}

The authors gratefully acknowledge the FASAL (Forecasting Agricultural output using Space, Agro-meteorology and Land based observations) Programme of Mahalanobis National Crop Forecast Centre (MNCFC), New Delhi, an Attached Office under Dept. of Agriculture, Cooperation \& Farmers Welfare, Ministry of Agriculture \& Farmers Welfare, Government of India (https://www.ncfc.gov.in/fasal.html) for sharing the crop information over the Karnataka state.

\section{REFERENCES}

Belgiu, M., \& Csillik, O. (2018). Sentinel-2 cropland mapping using pixel-based and object-based time-weighted dynamic time warping analysis. Remote Sensing of Environment, 204, 509523
Eitel, J.U., Vierling, L.A., Litvak, M.E., Long, D.S., Schulthess, U., Ager, A.A., Krofcheck, D.J., \& Stoscheck, L. (2011). Broadband, red-edge information from satellites improves early stress detection in a New Mexico conifer woodland. Remote Sensing of Environment, 115, 3640-3646

Forkuor, G., Dimobe, K., Serme, I., \& Tondoh, J.E. (2018). Landsat- 8 vs. Sentinel-2: examining the added value of sentinel2's red-edge bands to land-use and land-cover mapping in Burkina Faso. GIScience \& Remote Sensing, 55, 331-354

Jia, K., Wei, X., Gu, X., Yao, Y., Xie, X., \& Li, B. (2014). Land cover classification using Landsat 8 operational land imager data in Beijing, China. Geocarto International, 29, 941-951

Korhonen, L., Packalen, P., \& Rautiainen, M. (2017). Comparison of Sentinel-2 and Landsat 8 in the estimation of boreal forest canopy cover and leaf area index. Remote Sensing of Environment, 195, 259-274

Mandanici, E., \& Bitelli, G. (2016). Preliminary comparison of Sentinel-2 and Landsat 8 Imagery for a combined use. Remote Sensing, 8, 1014

Momeni, R., Aplin, P., \& Boyd, D.S. (2016). Mapping complex urban land cover from spaceborne imagery: the influence of spatial resolution, spectral band set and classification approach. Remote Sensing, 8, 88

Mountrakis, G., Im, J., \& Ogole, C. (2011). Support vector machines in remote sensing: A review. ISPRS Journal of Photogrammetry and Remote Sensing, 66, 247-259

Muller-Wilm, U., Louis, J., Richter, R., Gascon, F., \& Niezette, M. (2013). Sentinel-2 level 2A prototype processor: Architecture, algorithms and first results. In, Proceedings of the 2013 ESA Living Planet Symposium, Edinburgh, UK (pp. 9-13)

Novelli, A., Aguilar, M.A., Nemmaoui, A., Aguilar, F.J., \& Tarantino, E. (2016). Performance evaluation of object based greenhouse detection from Sentinel-2 MSI and Landsat 8 OLI data: A case study from Almería (Spain). International Journal of Applied Earth Observation and Geoinformation, 52, 403-411 
Pal, M., \& Mather, P. (2005). Support vector machines for classification in remote sensing. International Journal of Remote Sensing, 26, 1007-1011

Paul, S., \& Kumar, D.N. (2018). Spectral-spatial classification of hyperspectral data with mutual information based segmented stacked autoencoder approach. ISPRS Journal of Photogrammetry and Remote Sensing, 138, 265-280

Shao, Y., \& Lunetta, R.S. (2012). Comparison of support vector machine, neural network, and CART algorithms for the landcover classification using limited training data points. ISPRS Journal of Photogrammetry and Remote Sensing, 70, 78-87

Sharma, A., \& Mehrotra, R. (2014). An information theoretic alternative to model a natural system using observational information alone. Water Resources Research, 50, 650-660

Sharma, A., Mehrotra, R., Li, J., \& Jha, S. (2016). A programming tool for nonparametric system prediction using Partial Informational Correlation and Partial Weights. Environmental Modelling \& Software, 83, 271-275

Shoko, C., \& Mutanga, O. (2017). Examining the strength of the newly-launched Sentinel 2 MSI sensor in detecting and discriminating subtle differences between $\mathrm{C} 3$ and $\mathrm{C} 4$ grass species. ISPRS Journal of Photogrammetry and Remote Sensing, 129, 32-40

Sonobe, R., Yamaya, Y., Tani, H., Wang, X., Kobayashi, N., \& Mochizuki, K.-i. (2018). Crop classification from Sentinel-2derived vegetation indices using ensemble learning. Journal of Applied Remote Sensing, 12, 026019

Ustuner, M., Sanli, F., Abdikan, S., Esetlili, M., \& Kurucu, Y. (2014). Crop type classification using vegetation indices of RapidEye imagery. The International Archives of Photogrammetry, Remote Sensing and Spatial Information Sciences, 40, 195

Vermote, E., Justice, C., Claverie, M., \& Franch, B. (2016). Preliminary analysis of the performance of the Landsat 8/OLI land surface reflectance product. Remote Sensing of Environment, 185, 46-56

Zhang, H.K., Roy, D.P., Yan, L., Li, Z., Huang, H., Vermote, E., Skakun, S., \& Roger, J.-C. (2018). Characterization of Sentinel-2A and Landsat-8 top of atmosphere, surface, and nadir BRDF adjusted reflectance and NDVI differences. Remote Sensing of Environment 